\title{
Photoionization of $\mathrm{He}$ a Field Theoretic Approach
}

\author{
S. BhatTacharyYa* And S. Mitra \\ Gokhale Memorial Girls' College, Calcutta, India
}

(Received March 5, 2001; revised version July 10, 2001)

\begin{abstract}
The present paper deals with the field theoretic study of single ionization cross-sections $\sigma_{\mathrm{ph}}$ and $\sigma_{\mathrm{C}}$ of helium due to photoelectric effect and the Compton scattering, respectively, by photons of energy lying between $0-12 \mathrm{keV}$. In this energy range, $\sigma_{\mathrm{ph}}$ gradually decreases with energy while $\sigma_{\mathrm{C}}$ gradually increases. There is a crossover point around $6.5 \mathrm{keV}$ where $\sigma_{\mathrm{ph}}=\sigma_{\mathrm{C}}=10^{-24} \mathrm{~cm}^{2}$. Near about this crossover point $\sigma_{\mathrm{ph}}$ and $\sigma_{\mathrm{C}}$ curves are like mirror images. The ratio $\sigma_{\mathrm{C}} / \sigma_{\mathrm{ph}}$ lies between 0.3 to 1.5 in the energy range under consideration. The present results are compared with existing theoretical results.
\end{abstract}

PACS numbers: $32.80 . \mathrm{Cy}, 32.80 . \mathrm{Fb}$

\section{Introduction}

Morgan and Barlett [1], and Hino et al. [2] theoretically investigated single photoionization (SPI) by applying many-body perturbation theory (MBPT). Hino et al. [3] extended this approach to obtain cross-sections at high energies. There is also theoretical calculation by Andersson and Burgdorfer [4] on SPI by photoelectric effect (PEE). Most of the work done are related to the calculations of the ratio of double to single ionization by photon [3]. However, the calculations vary among themselves. Since there is still scope for theorisation, we have calculated here SPI of helium by PEE and the Compton scattering (CS) using field theory, Feynman diagram and covariant Lorentz gauge [5-8]. The Feynman diagram (Fig. 1) represents SPI due to PEE, where the high-energy photon interacts with the bound-electron cloud and ionizes it leaving only the $\mathrm{He}^{+}$ion in $1 s$-state. A change in the static correlation between the two bound electrons due to photon impact triggers the process of SPI. In the case of CS there are two Feynman diagrams (Fig. 2a and b)

*corresponding author; e-mail: sbgcmr@cal2.vsnl.net.in 
Fig. 1
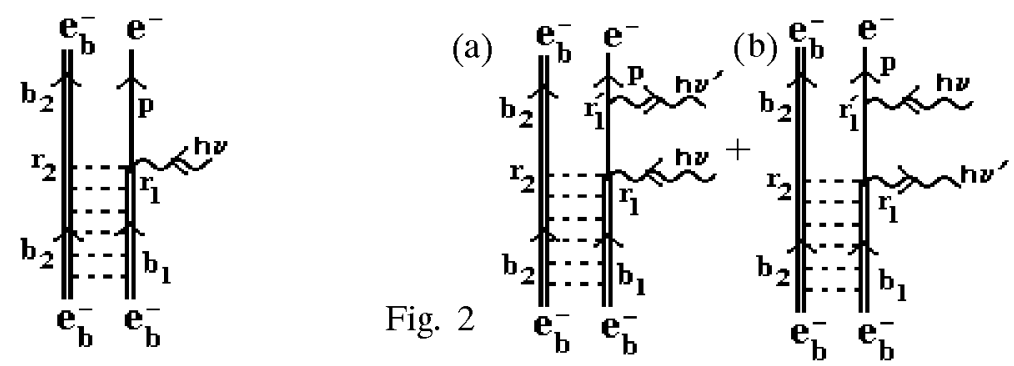

Fig. 1. Feynman diagram for photoionization. Double line represents bound electron, single line represents free electron, broken line represents Coulomb photon, wavy line with arrow represents photon.

Fig. 2. Feynman diagram for Compton scattering, the same as in Fig. 1: (a) direct scattering, (b) exchange scattering.

corresponding to direct and exchange interactions, respectively. Here along with the change in the static correlation between the two bound electrons, dynamic correlation through electron propagator between the bound and the corresponding outgoing electron come into play. In our calculations the cross-sections due to PEE gradually decrease with energy of the incident photon, while those due to CS increase. There is a crossover point near $6.5 \mathrm{keV}$, where cross-sections for both processes are the same. It is interesting to note that the Compton curve is mirror image of the photoelectric curve about this crossover point. Although the present $\sigma_{\mathrm{ph}}$ results deviate slightly from the existing theoretical results, a good agreement is obtained in the case of $\sigma_{\mathrm{C}}$.

\section{Mathematical formalism}

\subsection{Photoelectric effect}

The reaction for SPI by PEE is

$$
\mathrm{He}\left(1 s^{2}\right)+h \nu \rightarrow \mathrm{He}^{+}(1 s)+\mathrm{e} .
$$

The amplitude for the process is obtained from Fig. 1 and is given by $R_{p}$ [5]:

$$
R_{p}=\int \bar{\Psi}_{\mathrm{f}}\left(r_{1}, r_{2}, R\right) \gamma_{\mu} A_{\mu}\left(r_{1}, k\right) \Psi_{\mathrm{i}}\left(r_{1}, r_{2}, R\right) \mathrm{d}^{4} r_{1} \mathrm{~d}^{4} r_{2} \mathrm{~d} R,
$$

where $\gamma_{\mu}$ is the Dirac matrix and $A_{\mu}\left(r_{1}, k\right)$ is the wave function for transverse photon at $r_{1}$ with momentum $k . r_{1}, r_{2}$, and $R$ are the four-coordinates of the two bound electrons and the nucleus, respectively. The initial wave function of the system of bound electrons in He atom is given by [5-7]:

$$
\begin{gathered}
\Psi_{\mathrm{i}}\left(r_{1}, r_{2}, R\right)=\int \phi_{1 s^{2}}(x, y) \frac{1}{(2 \pi)^{3}} \frac{m}{\sqrt{b_{10} b_{20}}} u\left(b_{1}, r_{1}\right) u\left(b_{2}, r_{2}\right) \\
\times \exp (\mathrm{i} R L) \exp \left(\mathrm{i} b_{1} r_{1}+\mathrm{i} b_{2} r_{2}\right) \mathrm{d}^{4} r_{1} \mathrm{~d}^{4} r_{2} \mathrm{~d} R^{4},
\end{gathered}
$$


where $u\left(b_{i}, r_{i}\right)$ is the Dirac spinor for the bound electron with four-momenta $b_{i}$ at the position $r_{i}(i=1,2)$. $L$ and $L^{\prime}$ are the four-momenta of the nucleus in the initial and in the final state, respectively. The space coordinates of the electrons relative to the nucleus are

$$
\begin{aligned}
& x=r_{1}-\boldsymbol{R}, \quad y=r_{2}-\boldsymbol{R}, \\
& \Phi_{1 s^{2}}(x, y)=\chi_{1 s}(x) \chi_{1 s}(y) .
\end{aligned}
$$

For $\Phi_{1 s^{2}}$ we use the ground state correlated wave function of He atom as in [9]:

$$
\begin{aligned}
& \chi_{1 s}(x)=A \mathrm{e}^{-z_{1} x}+B \mathrm{e}^{-z_{2} x}, \\
& \quad A=0.7349, \quad B=0.799, \quad z_{1}=1.41, \quad z_{2}=2.61 .
\end{aligned}
$$

The final wave function of the system is

$$
\begin{aligned}
& \Psi_{\mathrm{f}}\left(r_{1}, r_{2}, R\right)=\frac{1}{\sqrt{V(2 \pi)^{3}}} \frac{m}{\sqrt{p_{0} b_{20}}} u(p) \mathrm{e}^{\mathrm{i}\left(r_{1} p\right)} \Phi_{1 s}^{\mathrm{He}^{+}}(y) \\
& \times u\left(b_{2} \mathrm{e}^{\mathrm{i} r_{2} b_{2}} \mathrm{e}^{\mathrm{i} R L^{\prime}} .\right.
\end{aligned}
$$

$L^{\prime}$ is the center of mass momentum of the interacting system in the final state. $E_{p}$ and $P$ are, respectively, the relativistic energy and momentum of the outgoing electron. The transverse potential $A_{\mu}\left(r_{1}, k\right)$ with photon polarization vectors $\lambda$ and momentum $k$ is given by

$$
A_{\mu}\left(r_{1}, k\right)=\frac{\lambda_{\mu} \mathrm{e}^{\mathrm{i} k r_{1}}}{\sqrt{2 k_{0} V(2 \pi)^{3}}} .
$$

$k$ is the four-momentum of the photon. On substituting (3)-(7) into Eq. (2)

$$
\begin{aligned}
R_{p}= & C \int\left[\bar{u}(p) \gamma_{\mu} \lambda_{\mu} u\left(b_{1}\right)\right]_{r_{1}}\left[\bar{u}\left(b_{2}\right) u\left(b_{2}\right)\right] r_{r_{2}} \mathrm{e}^{-\mathrm{i}\left(r_{1} p+r_{2} b_{2}+R L^{\prime}\right)} \\
& \times \mathrm{e}^{-\mathrm{i} k r_{1}} \mathrm{e}^{\mathrm{i}\left(b_{1} r_{1}+b_{2} r_{2}+R L\right)} \Phi_{1 s}^{\mathrm{He}^{+}}(y) \Phi_{1 s^{2}}(x, y) \mathrm{d}^{4} r_{1} \mathrm{~d}^{4} r_{2} \mathrm{~d}^{4} R
\end{aligned}
$$

Since at equal time $r_{10}=r_{20}=R_{0}$, after equal-time integration Eq. (8) becomes

$$
\begin{aligned}
R_{p}= & C \lambda_{\mu}\left[\bar{u}(p) \gamma_{\mu} u\left(b_{1}\right)\right]_{r_{1}}\left[\bar{u}\left(b_{2}\right) u\left(b_{2}\right)\right]_{r_{2}} 2 \pi \delta\left(p_{0}-k_{0}-b_{10}+L_{0}^{\prime}-L_{0}\right) \\
& \times \int \mathrm{e}^{-\mathrm{i}\left(r_{1} p+r_{2} b_{2}+R L^{\prime}\right)} \mathrm{e}^{-\mathrm{i} k r_{1}} \mathrm{e}^{\mathrm{i}\left(b_{1} r_{1}+b_{2} r_{2}+R L\right)} \\
& \times \Phi_{1 s}^{\mathrm{He}}(y) \Phi_{1 s^{2}}(x, y) \mathrm{d}^{3} r_{1} \mathrm{~d}^{3} r_{2} \mathrm{~d}^{3} R,
\end{aligned}
$$

where

$$
C=\frac{1}{\sqrt{V(2 \pi)^{3}}} \frac{m}{\sqrt{p_{0} b_{20}}} \frac{m}{\sqrt{b_{10} b_{20}}} \frac{1}{\sqrt{2 k_{0} V(2 \pi)^{3}}} \frac{1}{(2 \pi)^{3}} .
$$

The product of the spinors are

$$
T_{p 1}=\bar{u}(p) \gamma_{\mu} u\left(b_{1}\right), \quad T_{p 2}=\bar{u}\left(b_{2}\right) u\left(b_{2}\right)
$$


With

$$
T_{p}=T_{p 1} T_{p 2}
$$

we get

$$
R_{p}=C \lambda_{\mu} T_{p} 2 \pi \delta\left(p_{0}-k_{0}-b_{10}+L_{0}^{\prime}-L_{0}\right) I_{p},
$$

where $I_{p}$ is the overlap integral

$$
\begin{aligned}
I_{p}= & \int \mathrm{e}^{-\mathrm{i}\left(\boldsymbol{r}_{1} \cdot \boldsymbol{p}+\boldsymbol{r}_{2} \cdot \boldsymbol{b}_{2}+\boldsymbol{R} \cdot \boldsymbol{L}^{\prime}\right)} \mathrm{e}^{-\mathrm{i} \boldsymbol{k} \cdot \boldsymbol{r}_{1}} \mathrm{e}^{\mathrm{i}\left(\boldsymbol{b}_{1} \cdot \boldsymbol{r}_{1}+\boldsymbol{b}_{2} \cdot \boldsymbol{r}_{2}+\boldsymbol{R} \cdot \boldsymbol{L}\right)} \\
& \times \Phi_{1 s}^{\mathrm{He}^{+}}(y) \Phi_{1 s^{2}}(x, y) \mathrm{d}^{3} \boldsymbol{r}_{1} \mathrm{~d}^{3} \boldsymbol{r}_{2} \mathrm{~d}^{3} \boldsymbol{R} .
\end{aligned}
$$

On substituting $\boldsymbol{r}_{1}=\boldsymbol{x}+\boldsymbol{R}, \boldsymbol{r}_{2}=\boldsymbol{y}+\boldsymbol{R}$, and integrating over $x, y$

$$
\begin{aligned}
I_{p}= & (2 \pi)^{3} \delta^{3}\left(\boldsymbol{p}-\boldsymbol{k}-\boldsymbol{b}_{1}+\boldsymbol{L}^{\prime}-\boldsymbol{L}\right) I_{\mathrm{ph}}, \\
I_{\mathrm{ph}}= & {\left[\frac{64 A^{2} \pi^{2} z_{1}}{\left(z_{1}^{2}+t_{11}^{2}\right)^{2}\left(\alpha+z_{1}\right)^{3}}+\frac{8 A B \pi z_{1}}{\left(z_{1}^{2}+t_{11}^{2}\right)\left(\alpha+z_{2}\right)^{3}}\right.} \\
& \left.+\frac{8 A B \pi z_{2}}{\left(z_{2}^{2}+t_{11}^{2}\right)\left(\alpha+z_{1}\right)^{3}}+\frac{64 B^{2} \pi^{2} z_{2}}{\left(z_{2}^{2}+t_{11}^{2}\right)^{2}\left(\alpha+z_{2}\right)^{3}}\right],
\end{aligned}
$$

where

$$
\boldsymbol{t}_{11}=\boldsymbol{L}-\boldsymbol{L}^{\prime}=\boldsymbol{p}-\boldsymbol{k}-\boldsymbol{b}_{1} .
$$

Knowing that $\lambda^{2}=-1$, the square of the probability amplitude for PEE

$$
\left|M_{\mathrm{fi}}\right|^{2}=\left|R_{p} R_{p}^{*}\right|=C^{2}\left[(2 \pi)^{4} \delta^{4}\left(P-k-b_{1}+L^{\prime}-L\right)\right]^{2} I_{p}^{2} T_{p} T_{p}^{*} .
$$

Again from Refs. [10, 11]:

$$
\left[(2 \pi)^{4} \delta^{4}\left(P-k-b_{1}+L^{\prime}-L\right)\right]^{2}=V T(2 \pi)^{4} \delta^{4}\left(P-k-b_{1}+L^{\prime}-L\right),
$$

substituting

$$
\begin{aligned}
& P_{\mathrm{f}}-P_{\mathrm{i}}=p-k-b_{1}+L^{\prime}-L, \\
& E_{\mathrm{f}}-E_{\mathrm{i}}=E-k_{0}-b_{10}+L_{0}^{\prime}-L_{0},
\end{aligned}
$$

the transition probability per unit volume per unit time on using (15) and (15a) reads

$$
\begin{aligned}
W_{\mathrm{fi}} & =\frac{\left|M_{\mathrm{f}}\right|^{2}}{V T}=C^{2}(2 \pi)^{4} \delta^{4}\left(P_{\mathrm{f}}-P_{\mathrm{i}}\right) I_{\mathrm{ph}}^{2} T_{p} T_{p}^{*} \\
& =C^{\prime 2} \frac{1}{V^{2}} \frac{1}{k_{0}} \delta\left(E_{\mathrm{f}}-E_{\mathrm{i}}\right) \delta^{3}\left(\boldsymbol{P}_{\mathrm{f}}-\boldsymbol{P}_{\mathrm{i}}\right) I_{\mathrm{ph}}^{2} T_{p} T_{p}^{*} .
\end{aligned}
$$

To determine the cross-section for SPI due to PEE we have to divide $W_{\mathrm{fi}}$ by the flux of the incoming particles $\left|J_{\mathrm{inc}}\right|$ and the number of target particles per unit volume. 
$1 / V$ gives the latter, since the normalization of the wave functions was performed in such a way that there is just one particle in the normalization volume $V$. Furthermore, we have to sum (integrate) over the possible final states of the ionized electron and the final $\mathrm{He}^{+}$ion to obtain the cross-section. The number of final states in the momentum interval $\mathrm{d}^{3} p \mathrm{~d}^{3} L^{\prime}$ is

$$
V^{2} \frac{\mathrm{d}^{3} \boldsymbol{p}}{(2 \pi)^{3}} \frac{\mathrm{d}^{3} \boldsymbol{L}^{\prime}}{(2 \pi)^{3}}
$$

The cross-section is now given by the sixfold integration

$$
\sigma_{\mathrm{ph}}=\int \frac{1}{V} \frac{1}{\left|J_{\text {inc }}\right|} W_{\mathrm{fi}} V^{2} \frac{\mathrm{d}^{3} \boldsymbol{p}}{(2 \pi)^{3}} \frac{\mathrm{d}^{3} \boldsymbol{L}^{\prime}}{(2 \pi)^{3}} .
$$

As the target atom is assumed to be at rest $\boldsymbol{L}=\mathbf{0}$ and the incident flux becomes [11]:

$$
\left|J_{\text {inc }}\right|=\frac{1}{V}|c|, \quad c=\frac{|k|}{k_{0}} \quad \text { being the velocity of light. }
$$

This leads to

$$
\frac{1}{k_{0}} \frac{1}{V\left|J_{\text {inc }}\right|}=\frac{1}{k_{0}|c|}=\frac{1}{|k|} .
$$

$\mathrm{d}^{3} \boldsymbol{L}^{\prime}$ in (16) is integrated over momentum $\delta$-function and we get

$$
\boldsymbol{L}^{\prime}=\boldsymbol{k}-\boldsymbol{b}_{1}-p
$$

and we also use the relation [12]:

$$
\mathrm{d}^{3} p \delta\left(E_{\mathrm{f}}-E_{\mathrm{i}}\right)=\frac{|p|^{2}}{(\partial E / \partial|\boldsymbol{p}|)} \mathrm{d} \Omega=|\boldsymbol{p}| E \mathrm{~d} \Omega .
$$

Knowing that

$$
\begin{aligned}
& b_{1}=\left(b_{10}, 0\right), \quad b_{2}=\left(b_{20}, 0\right), \\
& T_{p} T_{p}^{*}=\left(T_{p 1} T_{p 1}^{*}\right)\left(T_{p 2} T_{p 2}^{*}\right),
\end{aligned}
$$

where

$$
\begin{aligned}
T_{p 1} T_{p 1}^{*} & =\left[\bar{u}(p) \gamma_{\mu} u\left(b_{1}\right)\right]\left[\bar{u}\left(b_{1}\right) \gamma_{\mu} u(p)\right], \\
T_{p 2} T_{p 2}^{*} & =\left[\bar{u}\left(b_{2}\right) u\left(b_{2}\right) \bar{u}\left(b_{2}\right) u\left(b_{2}\right)\right],
\end{aligned}
$$

we get after calculating the traces in (19)

$$
T_{p} T_{p}^{*}=\left[1-\frac{(m+E)\left(m-\varepsilon_{1 s}\right)}{m^{2}}\right]\left[\frac{\left(m-\varepsilon_{1 s}\right)^{2}}{m^{2}}+1\right] .
$$

The cross-section for PEE (16) becomes on using (17), (17a) and integrating over $\mathrm{d} \Omega$ :

$$
\sigma_{\mathrm{ph}}=\frac{4 \pi(2 \pi)^{4} C^{\prime 2}}{|k|} \frac{1}{(2 \pi)^{6}} T_{p} T_{p}^{*} I_{\mathrm{ph}}^{2}|\boldsymbol{p}| E .
$$


For small recoil momentum and small energy transfer to the ion we get from the energy $\delta$-function $E=k_{0}-\varepsilon_{1 s}$ :

$$
\sigma_{\mathrm{ph}}=\frac{(4 \pi)}{|\boldsymbol{k}|} \frac{C^{\prime 2}}{(2 \pi)^{6}} T_{p} T_{p}^{*} I_{\mathrm{ph}}^{2} m \sqrt{2 m}\left(k_{0}-\varepsilon_{1 s}\right)^{3 / 2} .
$$

Finally, the cross-section is obtained (Fig. 3) by assuming the recoil of the target to be very small: $t_{11} \approx 0$.

\section{D. Compton scattering}

The reaction for the process of SPI due to CS is given by

$$
\mathrm{He}+\gamma=\mathrm{He}^{+}(1 s)+\mathrm{e}^{-}+\gamma
$$

which is represented by two diagrams (Figs. $2 \mathrm{a}$, b). The second-order $S$-matrix $R_{\mathrm{CS}}$ for CS is given by current-current interactions. Contributions from the direct and exchange terms are, respectively, $R_{\mathrm{d}}, R_{\mathrm{e}}$, where

$$
\begin{aligned}
& R_{\mathrm{d}}=\left[\left(J_{\mu}^{\mathrm{e}^{-}}\right)_{r_{1}} A_{\mu}\left(k, r_{1}\right)\right]\left[\left(J_{\nu}^{\mathrm{e}^{-}}\right)_{r_{1}^{\prime}} A_{\nu}\left(k^{\prime}, r_{1}^{\prime}\right)\right] \\
& R_{\mathrm{e}}=\left[\left(J_{\mu}^{\mathrm{e}^{-}}\right)_{r_{1}^{\prime}} A_{\mu}\left(k, r_{1}^{\prime}\right)\right]\left[\left(J_{\nu}^{\mathrm{e}^{-}}\right)_{r_{1}} A_{\nu}\left(k^{\prime}, r_{1}\right)\right] .
\end{aligned}
$$

The electron currents at $r_{1}$ and $r_{1}^{\prime}$ are given by

$$
\begin{aligned}
& \left(J_{\mu}^{\mathrm{e}^{-}}\right)_{r_{1}}=\bar{\Psi}\left(r_{1}\right) \gamma_{\mu} \Psi\left(r_{1}, r_{2}, R\right), \\
& \left(J_{\mu}^{\mathrm{e}^{-}}\right)_{r_{1}^{\prime}}=\bar{\Psi}\left(r_{1}^{\prime}, r_{2}, R\right) \gamma_{\mu} \Psi\left(r_{1}^{\prime}\right),
\end{aligned}
$$

and the matrix element for SPI by CS is

$$
R_{\mathrm{CS}}=R_{\mathrm{d}}+R_{\mathrm{e}}
$$

(a) Direct term (Fig. 2a):

Using Eqs. (3)-(6) we write the following expression for the electron propagator between $r_{1}$ and $r_{1}^{\prime}$ :

$$
\Psi\left(r_{1}^{\prime}\right) \bar{\Psi}\left(r_{1}\right)=S\left(r_{1}^{\prime}-r_{1}\right)=\int \frac{\mathrm{e}^{-\mathrm{i} s\left(r_{1}^{\prime}-r_{1}\right)}(\hat{s}+m) \mathrm{d}^{4} s}{(2 \pi)^{4}\left(s^{2}-m^{2}\right)} .
$$

The direct term (24)

$$
\begin{aligned}
R_{\mathrm{d}}= & \int \bar{\Psi}\left(r_{1}^{\prime}, r_{2}, R\right) \gamma_{\nu} A_{\nu}\left(k^{\prime}, r_{1}^{\prime}\right) S\left(r_{1}^{\prime}-r_{1}\right) \\
& \times A_{\mu}\left(k, r_{1}\right) \gamma_{\mu} \Psi\left(r_{1}, r_{2}, R\right) \mathrm{d} r_{1} \mathrm{~d} r_{2} \mathrm{~d} r_{1}^{\prime} \mathrm{d} R .
\end{aligned}
$$

After necessary substitution

$$
R_{\mathrm{d}}=C_{\mathrm{c}} T_{\mathrm{d}} I_{\mathrm{d}}
$$

where $I_{\mathrm{d}}$ is the overlap integral, $T_{\mathrm{d}}$ is the product of the spinors and $C_{\mathrm{c}}$ is a constant, 


$$
\begin{aligned}
C_{\mathrm{c}}= & C \frac{1}{(2 \pi)^{4} \sqrt{2 V k_{0}^{\prime}(2 \pi)^{3}}}, \\
I_{\mathrm{d}}= & \int \frac{1}{s^{2}-m^{2}} \Phi_{1 s}^{\mathrm{He}}(y) \Phi_{1 s^{2}}(x, y) \exp \left\{\mathrm { i } \left[\left(r_{1}^{\prime} p+r_{2} b_{2}+R L^{\prime}\right)-k r_{1}\right.\right. \\
& \left.\left.-s^{\prime}\left(r_{1}^{\prime}-r_{1}\right)+k^{\prime} r_{1}^{\prime}-\left(b_{1} r_{1}+b_{2} r_{2}+R L\right)\right]\right\} \mathrm{d}^{4} r_{1} \mathrm{~d}^{4} r_{1}^{\prime} \mathrm{d}^{4} r_{2} \mathrm{~d}^{4} s^{\prime} .
\end{aligned}
$$

After equal-time integration and integration over $s^{\prime}$ as in (11)-(13) we get

$$
I_{\mathrm{d}}=I_{\mathrm{d}}^{\prime} \frac{1}{s_{t}^{2}-m^{2}} 2 \pi \delta_{0}\left(p_{0}+k_{0}^{\prime}-k_{0}-b_{10}+L_{0}^{\prime}-L_{0}\right)
$$

where $s_{t}=p+k^{\prime}$, and the overlap integral

$$
\begin{aligned}
I_{\mathrm{d}}^{\prime}= & N(2 \pi)^{3} \delta^{3}\left(\boldsymbol{p}+\boldsymbol{k}^{\prime}+\boldsymbol{L}^{\prime}-\boldsymbol{k}-\boldsymbol{L}\right) \\
& \times\left[\frac{64 A^{2} \pi^{2} z_{1}}{\left(z_{1}^{2}+\boldsymbol{t}_{1}^{2}\right)^{2}\left(\alpha+z_{1}\right)^{3}}+\frac{64 A B \pi^{2} z_{1}}{\left(z_{1}^{2}+\boldsymbol{t}_{1}^{2}\right)\left(\alpha+z_{2}\right)^{3}}\right. \\
& \left.+\frac{64 A B \pi^{2} z_{2}}{\left(z_{2}^{2}+\boldsymbol{t}_{1}^{2}\right)(\alpha+z)^{3}}+\frac{64 B^{2} \pi^{2} z_{2}}{\left(z_{2}^{2}+\boldsymbol{t}_{1}^{2}\right)^{2}\left(\alpha+z_{2}\right)^{3}}\right] .
\end{aligned}
$$

We substitute in the subsequent calculations

$$
\boldsymbol{t}_{1}=\boldsymbol{p}+\boldsymbol{k}^{\prime}-\boldsymbol{k}, \quad \boldsymbol{l}=\boldsymbol{L}-\boldsymbol{L}^{\prime}, \quad T_{\mathrm{d}}=T_{\mathrm{d} 1} T_{\mathrm{d} 2},
$$

where

$$
\begin{aligned}
T_{\mathrm{d} 1} & =\bar{u}(p) \gamma_{\mu} \lambda^{\mu}\left(\hat{s}_{t}+m\right) \gamma_{\nu} \lambda^{\nu} u\left(b_{1}\right) \\
T_{\mathrm{d} 2} & =\bar{u}\left(b_{2}\right) u\left(b_{2}\right)
\end{aligned}
$$

Since for the polarisation $\lambda, \lambda^{2}=-1$, we get after calculating the traces

$$
\begin{aligned}
& T_{\mathrm{d} 1} T_{\mathrm{d} 1}^{*}=\left[32\left(p \cdot \boldsymbol{s}_{t}\right) b_{10} s_{t 0}-16 p_{0} b_{10} s_{t}^{2}-16 m^{2}\left(p \cdot s_{t}\right)+16 m^{2} p_{0} b_{10}\right. \\
& \left.\quad-16 m^{2} s_{t 0} b_{10}+4 m^{2} s_{t}^{2}+m^{4}\right] \\
& T_{\mathrm{d} 2} T_{\mathrm{d} 2}^{*}=\frac{b_{2}^{2}}{m^{2}}+\frac{1}{4} \\
& R_{\mathrm{d}}=\frac{C_{\mathrm{c}} T_{\mathrm{d}} I_{\mathrm{d}}^{\prime}(2 \pi)^{4} \delta\left(E_{\mathrm{i}}-E_{\mathrm{f}}\right) \delta^{3}\left(\boldsymbol{P}_{\mathrm{i}}-\boldsymbol{P}_{\mathrm{f}}\right)}{s_{t}^{2}-m^{2}}
\end{aligned}
$$

and the square of the amplitude from direct term

$$
R_{\mathrm{d}}^{*} R_{\mathrm{d}}=\frac{C_{\mathrm{c}}^{2}\left[(2 \pi)^{4} \delta\left(E_{\mathrm{i}}-E_{\mathrm{f}}\right) \delta^{3}\left(\boldsymbol{P}_{\mathrm{i}}-\boldsymbol{P}_{\mathrm{f}}\right)\right]^{2} T_{\mathrm{d}}^{*} T_{\mathrm{d}} I^{\prime 2} \mathrm{~d}}{\left(s_{t}^{2}-m^{2}\right)^{2}}
$$


(b) Exchange term (Fig. 2b):

From (25) the exchange amplitude $R_{\mathrm{e}}$ is written as below:

$$
\begin{aligned}
R_{\mathrm{e}}= & \int \bar{\Psi}\left(r_{1}^{\prime}, r_{2}, R\right) \gamma_{\nu} A_{\nu}\left(k, r_{1}^{\prime}\right) S\left(r_{1}^{\prime}-r_{1}\right) \\
& \times A_{\mu}\left(k^{\prime}, r_{1}\right) \gamma_{\mu} \Psi\left(r_{1}, r_{2}, R\right) \mathrm{d} r_{1} \mathrm{~d} r_{2} \mathrm{~d} r_{1}^{\prime} \mathrm{d} R .
\end{aligned}
$$

After necessary substitution from (3)-(6) and (30) and writing

$$
\begin{aligned}
I_{\mathrm{e}}= & \int \frac{1}{s^{\prime 2}-m^{2}} \Phi_{1 s}^{\mathrm{He}}(y) \Phi_{1 s^{2}}(x, y) \exp \left\{\mathrm { i } \left[\left(r_{1}^{\prime} p+r_{2} b_{2}+R L^{\prime}\right)+k^{\prime} r_{1}\right.\right. \\
& \left.\left.-s^{\prime}\left(r_{1}^{\prime}-r_{1}\right)-k r_{1}^{\prime}-\left(b_{1} r_{1}+b_{2} r_{2}+R L\right)\right]\right\} \mathrm{d}^{4} r_{1} \mathrm{~d}^{4} r_{1}^{\prime} \mathrm{d}^{4} r_{2} \mathrm{~d}^{4} s^{\prime}, \\
T_{\mathrm{e} 1}= & \bar{u}(p) \gamma_{\mu} \lambda^{\mu}\left(\hat{s}_{\mathrm{e}}+m\right) \gamma_{\nu} \lambda^{\nu} u\left(b_{1}\right), \\
T_{\mathrm{e} 2}= & \bar{u}\left(b_{2}\right) u\left(b_{2}\right)=T_{\mathrm{d} 2}, \\
\text { and } T_{\mathrm{e}}= & T_{\mathrm{e} 1} T_{\mathrm{e} 2}, \text { the amplitude becomes } \\
R_{\mathrm{e}}= & C_{\mathrm{c}} T_{\mathrm{e}} I_{\mathrm{e}} .
\end{aligned}
$$

After equal-time integration and integration over $r_{1}^{\prime}, r_{1}, R$ and $s^{\prime}$ we get

$$
I_{\mathrm{e}}=2 \pi \delta_{0}\left(p_{0}+k_{0}^{\prime}-k_{0}-b_{10}+L_{0}^{\prime}-l_{0}\right) \frac{1}{s_{e}^{2}-m^{2}} I_{\mathrm{e}}^{\prime},
$$

where $s_{\mathrm{e}}=p-k$.

$$
\begin{aligned}
I_{\mathrm{e}}^{\prime}= & N(2 \pi)^{3} \delta^{3}\left(P_{\mathrm{i}}-P_{\mathrm{f}}\right)\left[\frac{64 A^{2} \pi^{2} z_{1}}{\left(z_{1}^{2}+\boldsymbol{t}_{1}^{2}\right)^{2}\left(\alpha+z_{1}\right)^{3}}+\frac{64 A B \pi^{2} z_{1}}{\left(z_{1}^{2}+\boldsymbol{t}_{1}^{2}\right)\left(\alpha+z_{2}\right)^{3}}\right. \\
& \left.+\frac{64 A B \pi^{2} z_{2}}{\left(z_{2}^{2}+\boldsymbol{t}_{1}^{2}\right)(\alpha+z)^{3}}+\frac{64 B^{2} \pi^{2} z_{2}}{\left(z_{2}^{2}+\boldsymbol{t}_{1}^{2}\right)^{2}\left(\alpha+z_{2}\right)^{3}}\right] \\
\boldsymbol{t}_{1}= & \boldsymbol{p}-\boldsymbol{k}-\boldsymbol{b}_{1} .
\end{aligned}
$$

Since $\lambda^{2}=-1$, we get for the trace parts

$$
\begin{aligned}
& T_{\mathrm{e} 1}^{*} T_{\mathrm{e} 1}=\left[32\left(\boldsymbol{p} \cdot \boldsymbol{s}_{\mathrm{e}}\right) b_{10} s_{\mathrm{e} 0}-16 p_{0} b_{10} s_{\mathrm{e}}^{2}\right. \\
& \left.\quad-16 m^{2}\left(\boldsymbol{p} \cdot \boldsymbol{s}_{\mathrm{e}}\right)+16 m^{2} p_{0} b_{10}-16 m^{2} s_{0} b_{10}+4 m^{2} s_{\mathrm{e}}^{2}+m^{4}\right], \\
& T_{\mathrm{e} 2}^{*} T_{\mathrm{e} 2}=T_{\mathrm{d} 2}^{*} T_{\mathrm{d} 2} .
\end{aligned}
$$

Finally, the square of the amplitude from exchange term becomes

$$
R_{\mathrm{e}}^{*} R_{\mathrm{e}}=\frac{C_{\mathrm{c}}^{2}\left[(2 \pi)^{4} \delta\left(E_{\mathrm{i}}-E_{\mathrm{f}}\right) \delta^{3}\left(\boldsymbol{P}_{\mathrm{i}}-\boldsymbol{P}_{\mathrm{f}}\right)\right]^{2} T_{\mathrm{e}}^{*} T_{\mathrm{e}} I_{\mathrm{e}}^{\prime 2}}{\left(s_{\mathrm{e}}^{2}-m^{2}\right)^{2}} .
$$




\subsection{Cross-section}

The total amplitude for SPI by the CS process is given by

$$
\left|M_{\mathrm{fi}}^{\mathrm{c}}\right|^{2}=R_{\mathrm{d}}^{*} R_{\mathrm{d}}+R_{\mathrm{e}}^{*} R_{\mathrm{e}}+R_{\mathrm{d}}^{*} R_{\mathrm{e}}+R_{\mathrm{e}}^{*} R_{\mathrm{d}}
$$

The interference of the direct and exchange term

$$
R_{\mathrm{d}}^{*} R_{\mathrm{e}}+R_{\mathrm{e}}^{*} R_{\mathrm{d}}=2 R_{\mathrm{d}}^{*} R_{\mathrm{e}}
$$

where

$$
R_{\mathrm{d}}^{*} R_{\mathrm{e}}=C_{\mathrm{c}}^{2}\left[(2 \pi)^{4} \delta^{3}\left(\boldsymbol{P}_{\mathrm{i}}-\boldsymbol{P}_{\mathrm{f}}\right) \delta\left(E_{\mathrm{i}}-E_{\mathrm{f}}\right)\right]^{2} \frac{T_{\mathrm{d}}^{*} I_{\mathrm{d}}^{\prime}}{s_{t}^{2}-m^{2}} \frac{T_{\mathrm{e}} I_{\mathrm{e}}^{\prime}}{s_{\mathrm{e}}^{2}-m^{2}} .
$$

Again from (34) and (40)

$$
\begin{aligned}
& T_{\mathrm{d}}^{*} T_{\mathrm{e}}=\left[T_{\mathrm{d} 1}^{*} T_{\mathrm{e} 1}\right]\left[T_{\mathrm{d} 2}^{*} T_{\mathrm{e} 2}\right] \\
& T_{\mathrm{d} 1}^{*} T_{\mathrm{e} 1}=\left(1 / 4 m^{2}\right)\left[p s_{\mathrm{d}}\left(16 b_{10} s_{\mathrm{e} 0}-8 m^{2}\right)+p s_{\mathrm{e}}\left(16 b_{10} s_{\mathrm{d} 0}-8 m^{2}\right)\right. \\
& \left.\quad+s_{\mathrm{d}} s_{\mathrm{e}}\left(4 m^{2}-16 p_{0} b_{10}\right)+16 m^{2} p_{0} b_{10}-8 m^{2} s_{\mathrm{d} 0} b_{10}-8 m^{2} s_{\mathrm{e} 0} b_{10}+4 m^{4}\right] \\
& \quad T_{\mathrm{d} 2}^{*} T_{\mathrm{e} 2}=\frac{b_{2}^{2}}{m^{2}}+\frac{1}{4}
\end{aligned}
$$

The transition probability per unit volume per unit time is

$$
W_{\mathrm{fi}}^{\mathrm{c}}=\frac{\left|M_{\mathrm{fi}}^{\mathrm{c}}\right|^{2}}{V T} \text {. }
$$

The cross-section for single ionization by the Compton scattering is given as in (16) by

$$
\sigma_{\mathrm{C}}=\int \frac{1}{V} \frac{1}{\left|J_{\text {inc }}\right|} W_{\mathrm{fi}}^{\mathrm{c}} V^{2} \frac{\mathrm{d}^{3} k^{\prime}}{(2 \pi)^{3}} \frac{\mathrm{d}^{3} p}{(2 \pi)^{3}} .
$$

Integration over $k^{\prime}$ is done using momentum $\delta$-function. Here we made use of (15a), (15b) and (17). The total cross-section is plotted in Fig. 3. In Fig. 4, ratio of the cross-sections by CS and PEE is shown.

\section{Results and discussions}

In this paper we have obtained single ionization of helium due to photoelectric effect and the Compton scattering in the energy range $0.1 \mathrm{keV}$ to $12 \mathrm{keV}$. Results are compared with the existing theoretical calculations [2, 4]. A comparative study of the two processes shows that in the energy region $0.5 \mathrm{keV}, \sigma_{\mathrm{ph}}$ dominates the ionization process over $\sigma_{\mathrm{C}}$ (Fig. 3). This is also found in the theoretical work of Hino et al. [2]. Beyond that energy $\sigma_{\mathrm{C}}$ is greater than $\sigma_{\mathrm{ph}}$. We have calculated single photoionization cross-section by PEE for helium from diagram (Fig. 1). It is a first-order process in which one of the bound electrons escapes due to a change in the static correlation between bound electrons by interaction with 


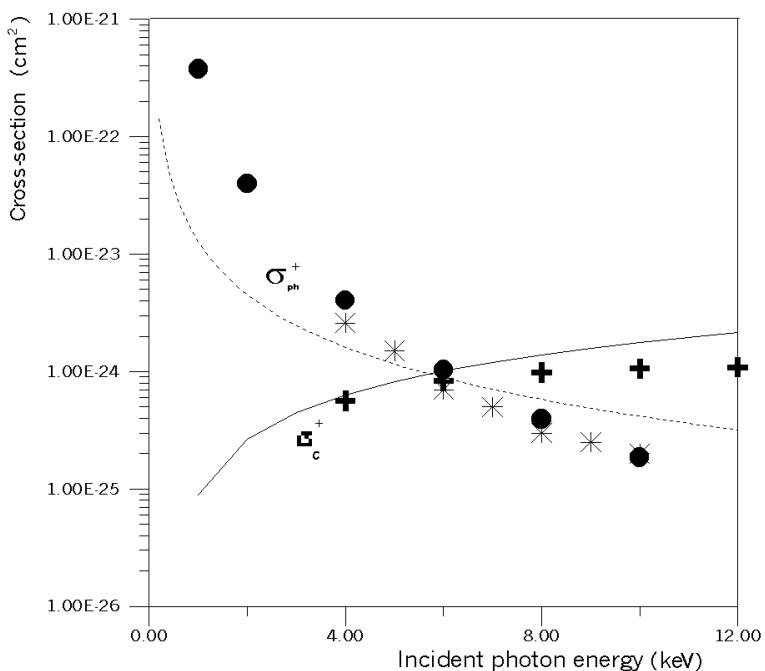

Fig. 3. Cross-section for single photoionization versus incident photon energy. Photoionization: present field theoretic calculation - - - -, calculation done by Andersson and Burgdorfer [4] *, MBPT [2] calculation by Hino et al. •. Compton scattering: present field theoretic calculation - , calculation by Hino et al. [3] + .

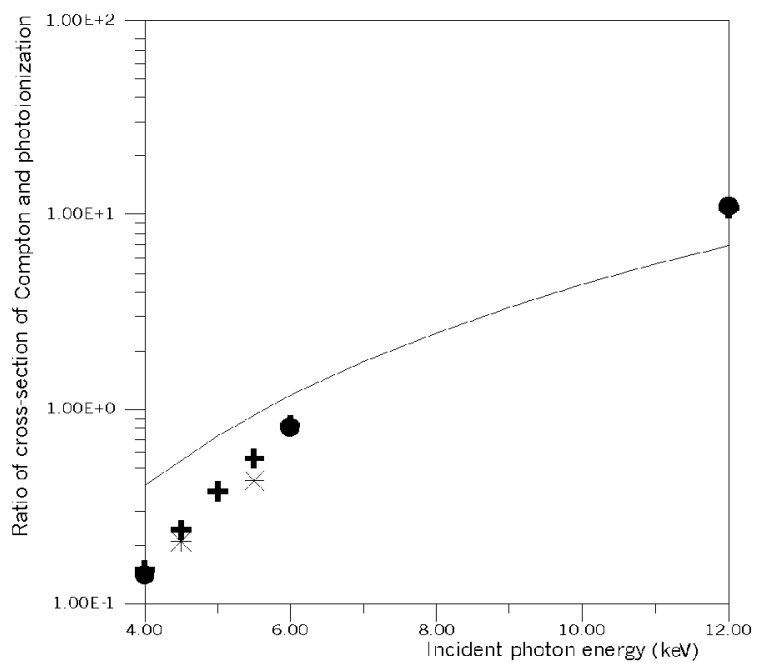

Fig. 4. Ratio of Compton scattering cross-section and photoionization cross-section versus incident photon energy. Present field theoretic calculation --, calculation by Viegele [13] +, calculation by Hino and McGuire [3] •, calculation by Morgan and Barlett [1]*.

photon, while the second electron remains bound with the helium ion in $1 s$-state. In the Compton scattering the Feynman diagrams are of second order and con- 
sist of direct and exchange diagrams. Physically CS is caused by change in static correlation along with the involvement of dynamic correlation between the bound and the ionized electron through photon propagator. Contributions from the direct and the exchange diagrams are small compared to the contribution from the interference term between the direct and exchange processes. The energy range of incident photon is $0.2-12 \mathrm{keV}$. In the case of photoelectric effect the cross-section asymptotically decreases with energy as in the case of Hino et al. [2]. Present value for $\sigma_{\mathrm{ph}}$ up to about $5 \mathrm{keV}$ is slightly below that of the existing theoretical value (Fig. 3), while the present result above $5 \mathrm{keV}$ remains slightly greater than the existing result. In the case of $\sigma_{\mathrm{C}}$, the present result almost agrees with the existing calculation (Fig. 3). However, $\sigma_{\mathrm{C}}$ rapidly increases with energy in the low energy region and the rate of increment becomes slow as energy increases from $7-12 \mathrm{keV}$. At about $6.5 \mathrm{keV}$ both the processes give similar contribution and beyond that energy the rate of increase in $\sigma_{\mathrm{C}}$ and rate of decrease in $\sigma_{\mathrm{ph}}$ are almost identical. It is interesting to note that the Compton curve is a mirror image of the photoelectric curve about this crossover point. The ratio of the ionization cross-sections (Fig. 4) by the Compton effect to that by photoelectric effect gradually increases with energy of the incident photon. Near $4 \mathrm{keV}$ it is almost 0.4 while near $6.5 \mathrm{keV}$ it is almost 1 . Beyond $7 \mathrm{keV}$ the ratio increases and becomes 1.4 near about $12 \mathrm{keV}$. In the high energy limit the Compton scattering dominates the ionization process.

\section{Conclusion}

In single ionization by the Compton scattering a part of the energy $K_{0}-\varepsilon_{1 s}$ is carried away by photoelectron and the remaining part by scattered photon, while in the photoelectric effect most of the energy $K_{0}-\varepsilon_{1 s}$ is carried away by photoelectron. Hence looking to the energy spectrum of the electrons one may diagnose which of the processes PEE and CS are involved in producing SPI in He.

In conclusion we find it most encouraging that this compact yet simple QED formulation can elegantly explain single photoionization by photoelectric effect and the Compton effect. The formulation of this photon-atom collision problem in a gauge-invariant language, which is similar to those describing most of the fundamental interactions in nature, has its own intrinsic appeal.

\section{Acknowledgments}

The work is supported by UGC, New Delhi through the project no. F10-17/98(SR-I). 


\section{References}

[1] D.V. Morgan, R.J. Barlett, Phys. Rev. A 59, 4075 (1999).

[2] K. Hino, P.M. Bergstrom, J.H. Macek, Phys. Rev. Lett. 72, 1620 (1994).

[3] K. Hino, T. Ishihara, F. Shimizu, N. Toshima, J.H. McGuire, Phys. Rev. A 48, 1271 (1993).

[4] L.R. Andersson, J. Burgdorfer, Phys. Rev. Lett. 71, 50 (1993).

[5] S. Bhattacharyya, S. Mitra, Phys. Rev. A 60, 2269 (1999).

[6] S. Bhattacharyya, S. Mitra, Phys. Rev. A 62,032709(1-7) (2000).

[7] S. Bhattacharyya, K. Pathak, Indian J. Phys. B 74, 1 (2000).

[8] S. Bhattacharyya, K. Pathak, Acta Phys. Pol. A 98, 335 (2000).

[9] S. Jetzke, F.H.M. Faisal, R. Hippler, H.O. Lutz, Z. Phys. A 315, 271 (1984).

[10] W. Greiner, J. Reinhardt, Quantum Electrodynamics, 2nd ed., Springer-Verlag, New York 1994

[11] C. Itzykson, J. Zuber, Quantum Field Theory, McGraw-Hill Book Co., New York 1980 .

[12] J.J. Sakurai, Advanced Quantum Mechanics, Addison Wesley, London 1967.

[13] W.T. Viegele, At. Data 5, 51 (1973). 